$\mathrm{T}$ he timing of surgical intervention for mitral regurgitation depends primarily on two factors: clinical symptoms; and the left ventricular response to volume overload. However, the aetiology of mitral regurgitation and the likelihood of surgical valve repair also are important factors in the optimal timing of surgical intervention.

\title{
AETIOLOGY AND DIAGNOSIS
}

The mitral valve apparatus is a complex anatomic and functional unit composed of the mitral annulus, valve leaflets, chordae, papillary muscles, and the underlying left ventricular wall. Normal function depends on both normal anatomy of each of these components and on the overall three dimensional relationships of these structures to each other, including the effects of overall left ventricular size, shape, and systolic function. Diverse mechanisms of mitral regurgitation are associated with different clinical outcomes. In addition, medical or surgical treatment is directed at the specific mechanism of regurgitation in each individual patient. Mitral regurgitation caused by an anatomic abnormality of the leaflets and chordae is termed primary regurgitation, while mitral regurgitation caused by a process primarily affecting the left ventricle is termed secondary mitral regurgitation. Examples of primary mitral regurgitation include myxomatous mitral valve disease which results in mitral regurgitation caused by leaflet prolapse and/or chordal rupture, rheumatic disease which typically causes increased leaflet stiffness with chordal shortening and fusion, and endocarditis with leaflet deformation and destruction. Examples of secondary mitral regurgitation include ischaemic disease that affects the function of the papillary muscles and underlying left ventricular wall, and dilated cardiomyopathy that alters the normal angle between the papillary muscles and mitral annulus (table 1).

Echocardiography allows reliable identification of the presence, severity and mechanism of mitral regurgitation. ${ }^{1}$ When transthoracic imaging is suboptimal, transoesophageal images provide excellent visualisation of mitral valve anatomy, and allow an accurate assessment of the aetiology of valve disease and the likelihood of surgical repair ( fig 1). The severity of mitral regurgitation can be quantitated by several approaches, including calculation of regurgitant volumes and fraction from calculation of volume flow rate at two intracardiac sites, from the proximal isovelocity surface area, or by measurement of the vena contracta on colour flow imaging. However, in practical terms, many clinicians rely on the simpler, albeit less quantitative, evaluation of regurgitation severity by colour flow imaging in multiple views (figs 2 and 3).

Assessment of the haemodynamic consequences of regurgitation is even more important than quantitation of regurgitant severity. Specific parameters that are important for clinical decision making are the degree of left ventricular dilation, left ventricular systolic function, left atrial enlargement, and pulmonary hypertension..$^{2-4}$

\section{PATHOPHYSIOLOGY AND CLINICAL OUTCOMES}

Primary mitral regurgitation often is initially diagnosed based on finding a systolic murmur in an asymptomatic patient. In some patients, the diagnosis is made only after the onset of atrial fibrillation or congestive heart failure symptoms. The clinical outcome of patients with symptomatic severe mitral regurgitation caused by primary mitral valve disease is very poor so that there is uniform agreement that valve surgery is indicated for symptoms due to primary mitral valve regurgitation.

In patients with primary mitral regurgitation, there may be an interval of several years between

Correspondence to: Catherine M Otto, MD, Division of Cardiology Box 356422, University of Washington, Seattle, WA 98195, USA:

cmotto@u.washington.edu the diagnosis of significant mitral regurgitation and onset of symptoms, with a rate of symptom onset of $2-4 \%$ per year in one series of 300 patients with mitral prolapse followed prospectively. ${ }^{5}$ However, the rate of symptom onset depends on the aetiology of mitral valve disease and the severity of regurgitation. For example, the rate of symptoms onset and adverse outcomes is much higher in patients with severe mitral regurgitation caused by a partial flail leaflet. ${ }^{6}$ In addition to development of symptoms, the two major concerns in patients with asymptomatic primary mitral valve disease are the risk of sudden death and the risk of irreversible left ventricular dysfunction. The risk 
Table 1 Mechanisms of mitral regurgitation

\begin{tabular}{ll}
\hline Disease process & Probable mechanism of regurgitation \\
\hline Myxomatous mitral valve disease & $\begin{array}{l}\text { Excess leaflet motion (chordal rupture, leaflet } \\
\text { prolapse) } \\
\text { Restricted leaflet motion (chordal shortening and } \\
\text { fusion, increased leaflet stiffness) } \\
\text { Destruction of valve tissue }\end{array}$ \\
Endocarditis & $\begin{array}{l}\text { Flail papillary muscle and leaflet } \\
\text { Coronary artery disease }\end{array}$ \\
$\begin{array}{l}\text { Infarction of papillary muscle or inferior-posterior } \\
\text { LV wall }\end{array}$ & $\begin{array}{l}\text { Abnormal tethering of chordae and leaflet } \\
\text { Ischaemic of inferior-posterior LV wall }\end{array}$ \\
$\begin{array}{l}\text { End stage disease with LV dilation and systolic } \\
\text { dysfunction }\end{array}$ & $\begin{array}{l}\text { Intermittent MR caused by abnormal chordal tethering } \\
\text { annulus, annular dilation }\end{array}$ \\
Dilated cardiomyopathy & $\begin{array}{l}\text { Altered angle between papillary muscle and mitral } \\
\text { annulus }\end{array}$
\end{tabular}

$L V$, left ventricular, MR, mitral regurgitation.

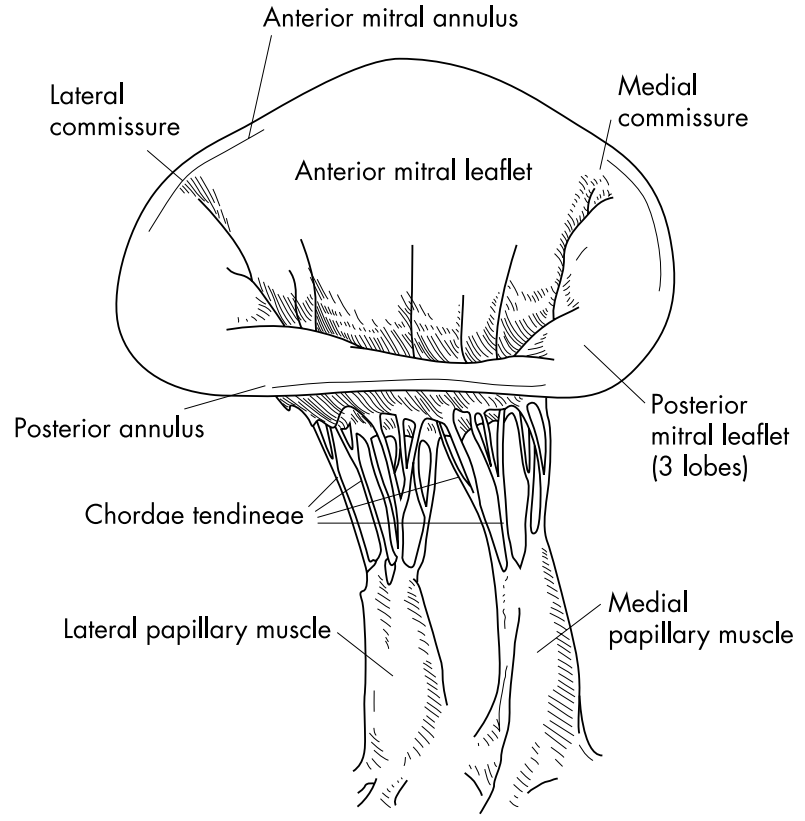

Figure 1 Normal function of the mitral valve apparatus depends on normal anatomy and function of the mitral annulus, the anterior and posterior leaflets, chordae, papillary muscles and the size, shape and systolic performance of the left ventricle. Reproduced with permission from: Otto CM. Valvular heart disease. Philadelphia: WB Saunders, 1999.

of sudden death is estimated to be 10 to 100 times normal, with an absolute risk of sudden death of $1-2.5 \%$ over six years. Risk factors for sudden death in patients with mitral regurgitation are left ventricular systolic dysfunction, leaflet redundancy, and severe mitral regurgitation. ${ }^{7}$ Unfortunately, it is unclear whether mitral valve surgery alters the risk of sudden death.

Chronic mitral regurgitation imposes a volume load on the left ventricle. In response to this volume load, initially the left ventricle empties more completely. Over time, left ventricular dilation maintains a normal forward cardiac output despite the regurgitant flow into the left atrium in systole. Early in the disease course, left ventricular systolic function remains normal, as measured by the rate of rise in ventricular pressure $(\mathrm{dP} / \mathrm{dt})$ or by end systolic elastance $\left(\mathrm{E}_{\max }\right)$. However, in the long term, some patients develop an irreversible decrease in left ventricular contractility. Diagnosis of impaired contractility is problematic as simple clinical measures of left ventricular per- formance, such as ejection fraction, may be in the normal range even when function is impaired, due to the altered loading conditions imposed by the regurgitant lesion itself. ${ }^{8}$ Of even more concern, this irreversible impairment in left ventricular function can occur in the absence of clinical symptoms. Thus, the clinical management of chronic asymptomatic mitral regurgitation focuses on periodic imaging studies for early detection of ventricular dysfunction.

Other adverse consequences of chronic mitral regurgitation that are related to the severity and duration of regurgitation include left atrial enlargement, atrial fibrillation, and pulmonary hypertension.

Secondary mitral regurgitation typically is diagnosed during evaluation of the primary disease process, often on echocardiography requested for evaluation of left ventricular function. Especially in patients with ischaemic mitral regurgitation, significant mitral regurgitation may not be audible on physical examination. In patients with secondary valve regurgitation, clinical outcome depends on the underlying disease process and it can be difficult to separate symptoms caused by mitral regurgitation from those caused by coronary or myocardial disease. In addition, treatment directed at the underlying disease process may favourably impact mitral regurgitation severity. For example, afterload reduction and optimisation of loading conditions in patients with dilated cardiomyopathy is associated with a significant decrease in mitral regurgitant severity, most likely because of improved alignment between the papillary muscles and mitral annulus. In patients with ischaemic mitral regurgitation, revascularisation to relieve ischaemia may be associated with a reduction in mitral regurgitant severity.

\section{MEDICAL TREATMENT}

In patients with chronic mitral regurgitation, medical treatment is directed at preventing the secondary complications of the disease process. Endocarditis prophylaxis is indicated based on accepted guidelines. In patients with rheumatic valve disease, guidelines for prevention of recurrent rheumatic fever also should be implemented. Since many patients with mitral regurgitation will eventually need surgical intervention, and since surgery is higher risk when coronary artery disease is present, it is especially important to evaluate and treat coronary artery disease risk factors. If atrial fibrillation occurs, treatment with anticoagulation and cardioversion or rate control is indicated.

Periodic clinical and echocardiographic evaluation is key to the optimal management of chronic mitral regurgitation. 

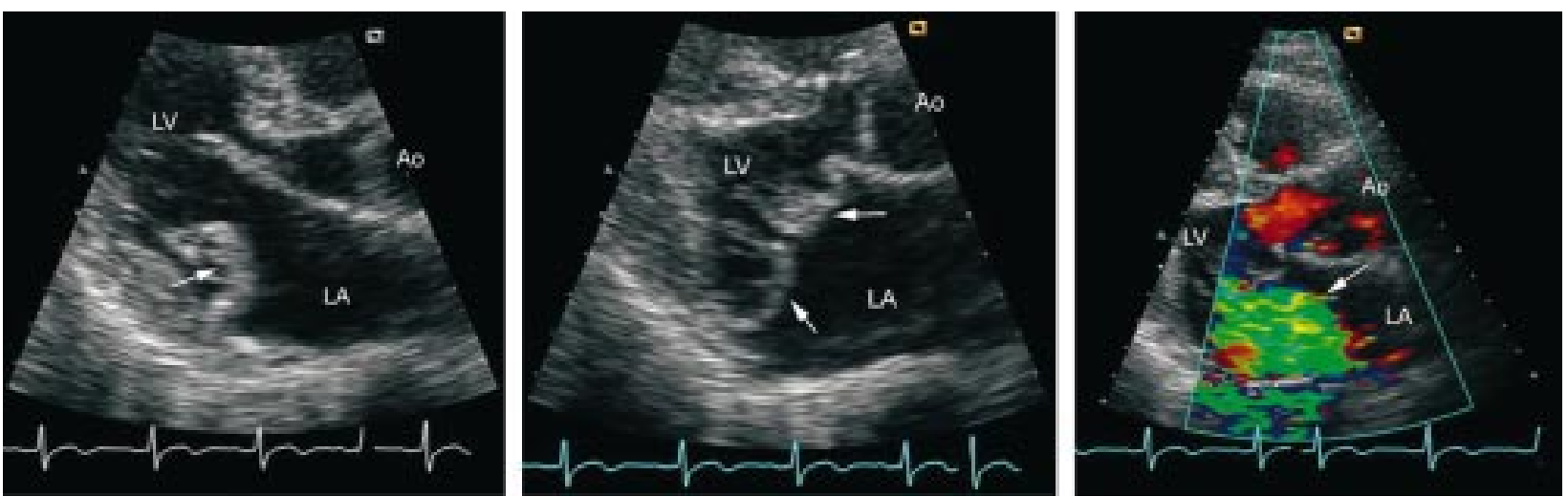

Figure 2 In a 55 year old man with an asymptomatic systolic murmur, echocardiographic images at end diastole (left) and end systole (centre) in a long axis view, show thickening and redundancy of the posterior mitral leaflet with prolapse of both leaflets into the left atrium in systole. Colour flow imaging (right) shows a large area of regurgitation. However, left ventricular size is normal with an end systolic dimension of $25 \mathrm{~mm}$, ejection fraction is $62 \%$, left atrial size is only mildly increased, and pulmonary pressures are normal. His physician is following the patient with annual echocardiography. Ao, aorta; LA, left atrium; LV, left ventricle.
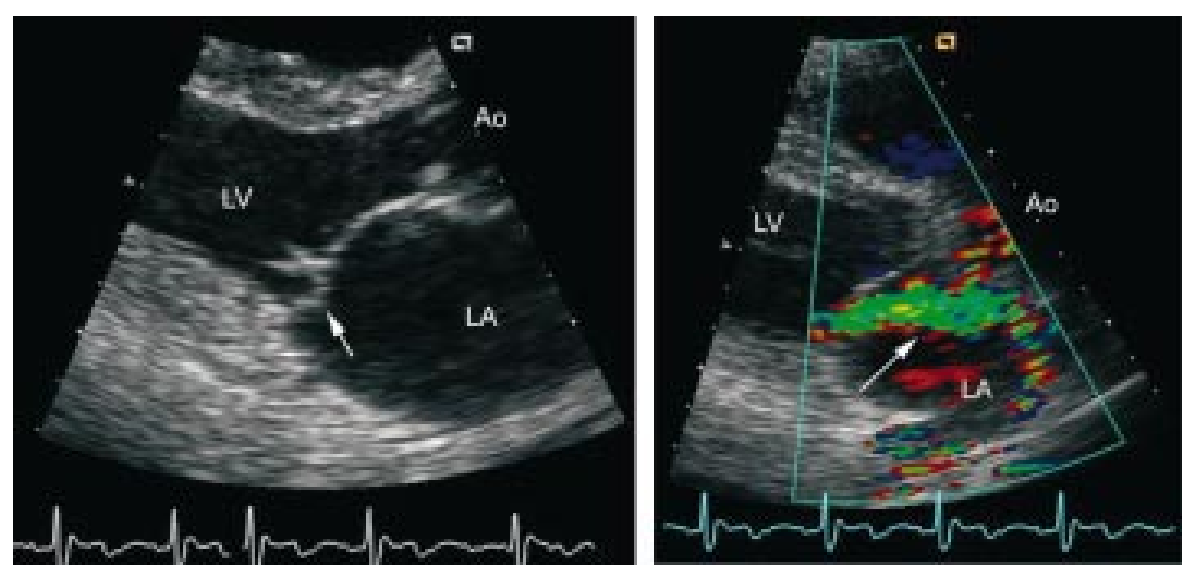

Figure 3 This 23 year old woman with recurrent postpartum atrial flutter and a systolic murmur has systolic prolapse of the posterior leaflet (left), although the leaflets are normal in thickness. There is an eccentric anteriorly directed jet of mitral regurgitation on colour flow imaging (right) consistent with posterior leaflet prolapse. Her left ventricular end systolic dimension, ejection fraction, and pulmonary pressures are normal. Left atrial size is mildly increased.

Prompt intervention at symptom onset and early intervention to prevent left ventricular systolic dysfunction or pulmonary hypertension provide optimal clinical outcomes. The frequency of echocardiography is determined by the cause and severity of mitral regurgitation. Patients with mild regurgitation need infrequent examinations, about every five years. Patients with moderate mitral regurgitation and normal left ventricular size are examined every 1-2 years. However, if regurgitation is severe or if "moderate" regurgitation is accompanied by left ventricular dilation, annual echocardiography is appropriate. The frequency of examination should be increased if there is an interim change in symptoms or physical examination, new onset atrial fibrillation, or if sequential studies suggest progressive left ventricular dilation, contractile dysfunction or increasing pulmonary pressures. Conversely, in a patient with mild or moderate regurgitation, the interval between echocardiograms may be lengthened if there are stable findings over two to three examination intervals.

There is no specific approach to the disease process itself in patients with primary mitral regurgitation. There are no known therapies that favourably alter the leaflet tissue in myxomatous mitral valve disease; the leaflet abnormalities in this genetic disorder appear intrinsic with no known associated clinical factors or medical treatment. In addition, medical treatment directed at prevention of left ventricular dilation is controversial. Although afterload reduction appears to favourably alter ventricular geometry and reduce regurgitant severity in patients with dilated cardiomyopathy, the haemodynamic effects are less clear in patients with primary mitral valve disease. ' Furthermore, if the left ventricular response to volume overload is a beneficial compensatory response, rather than a pathological response, prevention of ventricular adaptation may not improve clinical outcome. An additional concern is that medical treatment might "mask" the recognition of ventricular dysfunction, thus resulting in an unnecessary delay in surgical intervention.

\section{SURGICAL OPTIONS}

The surgical options for treatment of mitral regurgitation include mitral valve replacement, with or without chordal preservation, and mitral valve repair (fig 4). Mitral valve repair offers several advantages including avoidance of long term anticoagulation and, most importantly, preservation of the continuity between the mitral annulus and papillary muscles. This annular-papillary muscle continuity helps maintain normal left ventricular geometry and systolic function. When annular-papillary continuity is preserved, ejection fraction typically remains stable or improves after mitral surgery in contrast to a decline by an average of 10 ejection fraction units when this continuity is disrupted. ${ }^{10-12}$ Preservation of left ventricular dysfunction translates into decreased operative mortality and improved long term outcomes for repair versus replacement of the mitral valve. The average operative mortality for mitral valve repair is $1-2 \%$ compared to $5-10 \%$ with valve replacement. In addition, the mitral valve repair procedure is very durable with a low rate of reoperation for 

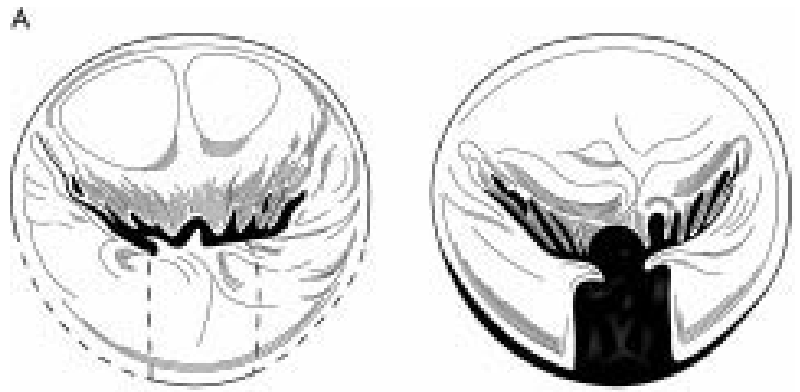

B

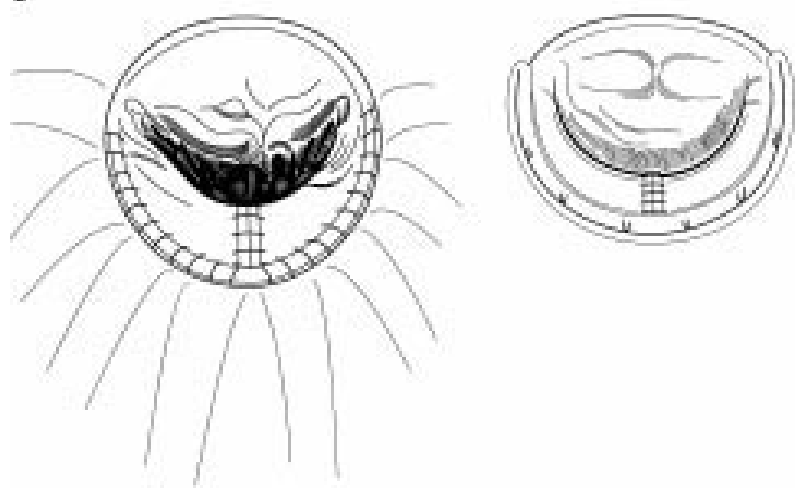

Figure 4 Schematic diagram of a mitral valve repair procedure. A quadrangular resection of the redundant or flail segment of the posterior leaflet is performed (A) and the posterior leaflet is detached from the annulus (sliding leaflet technique). The remaining posterior leaflet tissue is brought together, the resection is sutured, and the posterior leaflet is reattached to the annulus with placement of a flexible posterior annuloplasty ring (B). Reproduced with permission from: Gillinov AM, Cosgrove DM. Ann Thoracic Surgery 1999;68:2356-7.

recurrent mitral regurgitation, and with an event-free survival ranging from $80-90 \%$ at $5-10$ years in published series. ${ }^{13-17}$

When mitral valve repair is not feasible, the next option is mitral valve replacement with chordal preservation. Various surgical techniques are used to ensure the residual mitral valve tissue does not interfere with function of the valve prosthesis. Typically, a mechanical valve is used because of concerns about longevity of tissue valves in the mitral position and because many patients require long term anticoagulation for chronic atrial fibrillation in any case. Stented tissue valves are used when anticoagulation is contraindicated. There are preliminary reports proposing the use of a mitral valve homograft replacement, but this option is not widely available at this time.

Although the surgeon makes the final decision after direct inspection of the valve as to whether the valve can be repaired or must be replaced, echocardiography is highly reliable in determining whether valve repair is likely to be successful. On echocardiographic imaging, the key features that help predict the likelihood of valve repair are the thickness and redundancy of the anterior and posterior mitral valve leaflets, identification of prolapsing or flail leaflet segments with associated ruptured chordae, and the direction of the mitral regurgitant jet. It is important to evaluate the exact site of valve dysfunction; anterior leaflet repairs are more difficult than posterior leaflet repairs; the posterior leaflet has three scallops (medial, middle, and lateral); and an occasional patient has dysfunction localised to the medial or lateral commissural region. Many of
Table 2 Predictors of outcome after surgery for mitral regurgitation

Primary predictors

- Clinical symptoms caused by mitral regurgitation

- LV end systolic volume or dimension

- LV ejection fraction

Secondary factors

$\checkmark$ Comorbid disease

Coronary artery disease

Other valve involvement

- Other measures of LV

function

Wall stress

- Surgical procedure

LV maximum $\mathrm{dP} / \mathrm{dt}$

- Other sequelae of

Mitral valve replacement

Mitral valve repair

volume overload

Left atrial size

LV end diastolic pressure

Pulmonary artery pressure

Right ventricular ejection fraction

Atrial fibrillation

$L V$, left ventricular.

these features can be appreciated on a good quality transthoracic examination, but definitive diagnosis usually requires transoesophageal imaging for precise delineation of valve anatomy. Blood pressure and heart rate during the examination should be similar to the patient's normal state to ensure regurgitant severity is not underestimated. It is preferable that this examination be performed before scheduling surgery so that the cardiologist and surgeon can decide on the potential approaches and discuss these options with the patient.

\section{PREDICTION OF OUTCOMES AFTER MITRAL VALVE SURGERY}

In patients with symptomatic severe chronic mitral regurgitation and patients with acute severe mitral regurgitation, prompt surgical intervention is indicated for relief of symptoms and preservation of left ventricular systolic function. The difficult clinical decision is whether surgery is indicated in an asymptomatic patient with chronic severe mitral regurgitation. Since the patient feels well, there must be convincing evidence that surgery will prolong life or improve the long term quality of life. In addition, potential benefit must be greater than the risk and complications of surgery.

The current guidelines for optimal timing of surgical intervention in chronic mitral regurgitation have been derived from studies evaluating the effect of preoperative factors on long term outcome after surgical intervention. All of these series include only patients with "severe" mitral regurgitation; albeit the definition of severity varies from study to study and typically is based on clinical, not quantitative Doppler, criteria. There are no studies yet available showing the predictive value of quantitative measures of regurgitant severity in the timing of surgical intervention.

Instead, it is the physiologic consequences of chronic mitral regurgitation that provide the best predictors of clinical outcome-left ventricular size and systolic function, left atrial size, pulmonary artery pressures, and atrial fibrillation-with the left ventricular response to chronic volume overload having the strongest predictive value (table 2). From a theoretical point of view, the optimal measure of left ventricular function should reflect contractility and be independent of preload, afterload, and comorbid diseases. In research studies, measures such as left ventricular elastance derived from multiple pressure-volume loops have been used to approximate this ideal measure. 
Table 3 American College of Cardiology/American Heart Association guidelines for surgery for non-ischaemic severe mitral regurgitation

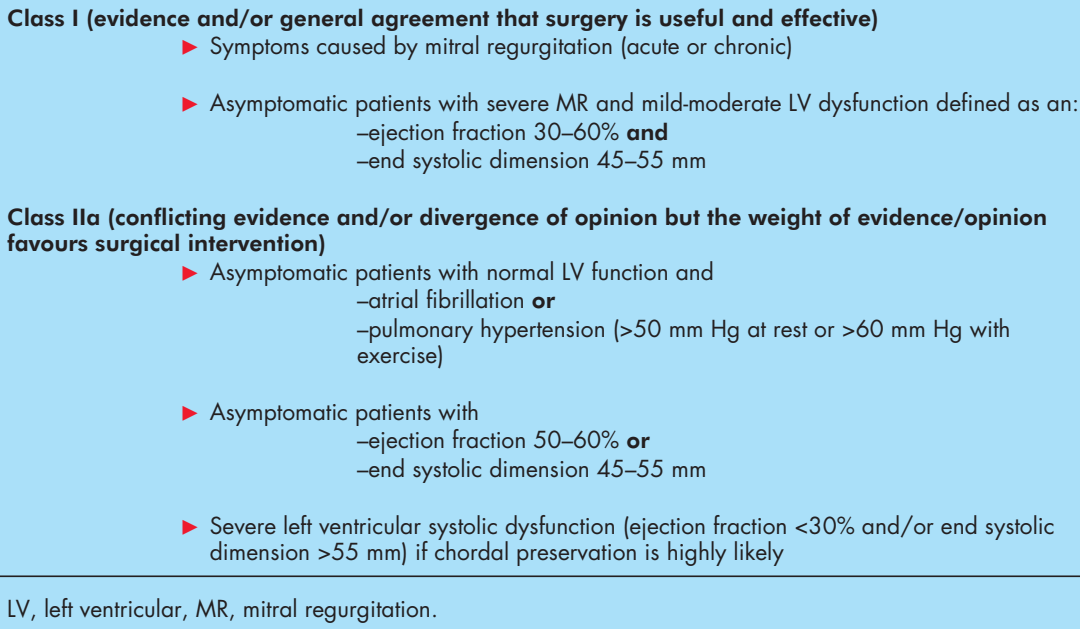

$L V$, left ventricular, $M R$, mitral regurgitation

In practical clinical terms, simpler measures such as left ventricular end systolic and end diastolic dimensions and ejection fraction have been used as these measures are easily obtained, reproducible, and predict clinical outcome, even though they are imperfect measures of ventricular contractility. As a general guideline, surgery should be considered in chronic asymptomatic severe mitral regurgitation when left ventricular end systolic dimension is $\geqslant 45 \mathrm{~mm}$ (normal $<40 \mathrm{~mm}$ ) and ejection fraction is $\leqslant 60 \%$ (normal $65 \%$ ). Since there is some variability in echocardiographic measurements, it is preferable to document a consistent trend on serial echocardiographic studies or to repeat a study after a shorter interval for abnormal values, rather than recommending surgery based on a single number. The current American College of Cardiology/American Heart Association guidelines for timing of surgery are shown in table 3.

In the operating room, predictors of long term outcome are the surgical procedure (repair versus replacement) and the degree of residual mitral regurgitation after valve repair as assessed by intraoperative transoesophageal echocardiography, with loading conditions matched to a baseline study performed at the beginning of the surgical procedure. A successful repair is associated with no or minimal mitral regurgitation. If more severe regurgitation persists, a second bypass pump run with repeat repair or valve replacement may be indicated, although this decision is modulated by other factors in individual patients.

\section{CONTROVERSIES}

Several issues in the optimal timing of surgical intervention for chronic mitral regurgitation are unresolved because current guidelines are based on incomplete natural history data and on the consensus of experts, rather than on prospective randomised trials.

The first issue is the definition of severe mitral regurgitation; specifically should severe mitral regurgitation be defined by quantitative Doppler techniques or by the physiologic consequences of chronic volume overload? Some centres have proposed that patients with severe mitral regurgitation defined by a specific regurgitant volume or regurgitant orifice area should undergo earlier surgical intervention, particularly if valve repair is feasible. However, this approach assumes that all these patients will rapidly develop irreversible consequences of the valvar lesion, such as left ventricular dysfunction. It is worrisome that mitral valve prolapse is associated with an increased risk of sudden death, but there are no convincing data that surgical intervention decreased this risk. Until we have prospective data supporting the hypothesis that early intervention decreases the adverse consequences of chronic mitral regurgitation, mitral valve surgery based on Doppler criteria alone is not appropriate.

The utility and timing of surgery for secondary mitral regurgitation remains controversial. In patients with ischaemic mitral regurgitation, revascularisation alone may result in a decrease in regurgitant severity if the aetiology was intermittent ischaemia. In other patients, significant regurgitation persists after revascularisation; in this subgroup, a mitral annuloplasty to reduce regurgitant severity may be beneficial. In the absence of controlled clinical trials, the decision to repair the mitral valve in patients with ischaemic mitral regurgitation is based on specific factors in each case and there is wide variability in the use of this approach between institutions. Mitral valve repair in patients with regurgitation secondary to dilated cardiomyopathy is even more controversial. Some centres report improved outcomes with mitral repair in this group of patients, but this approach has not been widely accepted.

In the patient with mitral regurgitation and atrial fibrillation, some centres now advocate a concurrent atrial procedure to restore sinus rhythm and prevent recurrent atrial fibrillation. As the surgical procedure to isolate the atrial tissue has become simpler, this procedure is likely to become used more widely and likely will be a standard adjunct to mitral valve repair in the future.

The other problematic area in clinical decision making in patients with severe mitral regurgitation is whether it is ever "too late" for surgical intervention. In patients who first present with severe left ventricular dilation and systolic dysfunction caused by chronic volume overload, outcome after mitral valve replacement is poor. In patients with severe mitral regurgitation and an ejection fraction $<30 \%$ or an end systolic dimension $>55 \mathrm{~mm}$, surgery should be considered only if it is highly likely that a valve repair can be performed. 
Timing of surgery in mitral regurgitation: key points

- Primary mitral regurgitation is caused by an anatomic abnormality of the valve apparatus while secondary mitral regurgitation is caused by abnormal three dimensional relationships between valve components

- Mitral valve surgery is indicated for symptoms caused by severe mitral regurgitation

- Chronic left ventricular volume overload may lead to an irreversible decline in contractility, even in the absence of symptoms

- Practical clinical measures for early contractile dysfunction are a left ventricular end systolic dimension $\geqslant 45 \mathrm{~mm}$ and an ejection fraction $\leqslant 60 \%$

- Preservation of continuity between the mitral annulus and papillary muscles at the time of surgery helps maintain left ventricular systolic function

- Mitral valve repair has a lower operative mortality and better long term outcome than mitral valve replacement

\section{REFERENCES}

1 Otto CM. Valvular regurgitation: diagnosis, quantitation and clinical approach. In: Otto CM, ed. Textbook of clinical echocardiography, 2nd ed. Philadelphia: WB Saunders, 2000:265-300.

- Basic echocardiographic approach to evaluation of mitral valve anatomy and evaluation of regurgitant severity.

2 Bonow RO, Carabello BA, deleon AC, et al. ACC/AHA guidelines for the management of patients with valvular heart disease: a report of the American College of Cardiology/American Heart Association task force on practice guidelines (committee on management of patients with valvular heart disease). J Am Coll Cardiol 1998:32:1486-8.

- Consensus guidelines on the optimal management of patients with valvar heart disease. Concise summary of the literature and extensive list of references.

3 Otto $\mathrm{CM}$. Evaluation and management of chronic mitral regurgitation. N Engl J Med 2001;345:740-6.

- Recent review summarising the strategies and evidence for management of chronic mitral regurgitation. A flow chart summarising the ACC/AHA guidelines and 25 key references are included.

4 Meier DJ, Landolfo CL, Starling MR. The role of echocardiography in the timing of surgical intervention for chronic aortic and mitral regurgitation. In: Otto CM, ed. The practice of clinical echocardiography, 2nd ed. Philadelphia: WB Saunders, 2002.

- Detailed review of the evidence for determining the optimal timing of surgical intervention for mitral regurgitation. Practical algorithm for clinical use is provided.

5 Duren DR, Becker AE, Dunning AJ. Long-term follow-up of idiopathic mitral valve prolapse in 300 patients: a prospective study. J Am Coll Cardiol 1988;11:42-7.

- In this classic prospective study including patients with varying degrees of mitral regurgitation caused by mitral prolapse, the natural history of the disease was slow with a survival rate of $97 \%$ at six years.
6 Ling LH, Enriquez Sarano M, Seward JB, et al. Clinical outcome of mitral regurgitation due to flail leaflet. N Engl J Med 1996;335:1417-23.

- The rate of surgery or death in a series of 229 patients with severe mitral regurgitation was $90 \%$ at 10 years in patients with a flail leaflet segment identified on echocardiography. This paper emphasises the importance of valve anatomy and regurgitant severity as primary factors determining clinical outcome in patients with myxomatous mitral valve disease.

7 Grigioni F, Enriquez-Sarano $M$, Ling $\mathrm{LH}$, et al. Sudden death in mitral regurgitation due to flail leaflet. J Am Coll Cardiol 1999;34:2078-85.

- In 348 patients with a flail mitral leaflet and a mean follow up of four years, the rate of sudden death was related to NYHA functional class, ejection fraction, and atrial fibrillation. The annual rate of sudden death was $1 \%$ for patients in class $\mathrm{I}, 3.1 \%$ for those with class II symptoms, and $7.8 \%$ for those with class III/IV symptoms.

8 Flemming MA, Oral H, Rothman ED, et al. Echocardiographic markers for mitral valve surgery to preserve left ventricular performance in mitral regurgitation. Am Heart J 2000;140:476-82.

9 Levine HJ, Gaasch WH. Vasoactive drugs in chronic regurgitant lesions of the mitral and aortic valves. J Am Coll Cardiol 1996;28:1083-91.

- Review of the data on the acute and long term effects of vasodilator treatment for secondary valvar regurgitation. This review summarises the beneficial effects of medical treatment for chronic mitral regurgitation and highlights the controversies in the treatment of primary mitral regurgitation.

10 Rozich JD, Carabello BA, Usher BW, et al. Mitral valve replacement with and without chordal preservation in patients with chronic mitral regurgitation. Mechanisms for differences in postoperative ejection performance. Circulation 1992;86:1718-26.

- Classic paper demonstrating the effect of chordal preservation on left ventricular ejection fraction after mitral valve surgery. When the mitral chords are severed the left ventricle becomes more spherical.

11 Leung DY, Griffin BP, Stewart WJ, et al. Left ventricular function after valve repair for chronic mitral regurgitation: predictive value of preoperative assessment of contractile reserve by exercise echocardiography. J Am Coll Cardiol 1996;28:1198-205.

12 Enriquez Sarano M, Schaff HV, Orszulak TA, et al. Valve repair improves the outcome of surgery for mitral regurgitation. A multivariate analysis. Circulation 1995;91:1022-8.

- In 214 patients undergoing mitral valve replacement, mean (SD) ejection fraction dropped from 60 (12)\% to 49 (15)\%. In 195 patients undergoing mitral valve repair, ejection fraction went from $63(9 \%)$ to $54(11) \%$.

13 Enriquez Sarano M, Tajik AJ, Schaff HV, et al. Echocardiographic prediction of left ventricular function after correction of mitral regurgitation: results and clinical implications. J Am Coll Cardiol 1994;24:1536-43.

14 Crawford MH, Souchek J, Oprian CA, et al. Determinants of survival and left ventricular performance after mitral valve replacement. Department of Veterans Affairs cooperative study on valvular heart disease. Circulation 1990;81:1173-81.

15 Otto CM. Surgical intervention for mitral regurgitation. In: Otto CM Valvular heart disease. Philadelphia: WB Saunders, 1999

16 Lee EM, Shapiro LM, Wells FC. Importance of subvalvular preservation and early operation in mitral valve surgery. Circulation 1996;94:211723.

17 David TE, Armstrong S, Sun Z, et al. Late results of mitral valve repair for mitral regurgitation due to degenerative disease. Ann Thorac Surg 1993;56:7-12. 